\title{
Nonconforming methods for nonlinear elasticity problems *
}

\author{
Bernd Flemisch and Barbara I. Wohlmuth \\ University of Stuttgart, Institute for Applied Analysis and Numerical Simulation \\ \{flemisch, wohlmuth\}@ians. uni-stuttgart.de
}

Summary. Domain decomposition methods are studied for several problems exhibiting nonlinearities in terms of curved interfaces and/or underlying model equations. In order to retain as much flexibility as possible, we do not require the subdomain grids to match along their common interfaces. Dual Lagrange multipliers are employed to generate efficient and robust transmission operators between the subdomains. Various numerical examples are presented to illustrate the applicability of the approach.

\section{Introduction}

We apply domain decomposition techniques to efficiently discretize nonlinear elasticity problems. The framework of mortar methods, $[1,2,3,8]$, is employed to deal with nonmatching grids. Especially for the applications discussed in Section 3, we recommend the use of dual discrete Lagrange multiplier spaces as in [5]. They are a basic ingredient for the formulation and the performance of our numerical solution procedures presented there.

In Section 2, we focus on a type of nonlinearity arising only from the geometry of the subdomain interfaces, namely, when the interfaces are curved and therefore require a nonlinear parametrization. The subdomain grids originating from a nonoverlapping decomposition may now overlap or even exhibit gaps along the curved interface. Transferring the methodology of the scalar setting to elasticity problems, we encounter a preasymptotic misbehavior when using dual Lagrange multipliers on the coarse side and present a remedy.

Section 3 deals with nonlinear elasticity model equations. First, two-body contact problems are studied, where we use an inexact primal-dual active set strategy as our solution method. The last part is devoted to the geometrically nonlinear elasticity setting and to the use of Neo-Hooke materials.

^ This work was supported in part by the Deutsche Forschungsgemeinschaft, SFB 404, B8, C12. 


\section{Curvilinear boundaries}

Scalar case. For simplicity, we first restrict ourselves to the case of two 2D subdomains sharing a closed interface curve and refer to [4] for a complete analysis for many subdomains. We consider the model problem

$$
-\Delta u=f \text { in } \Omega \subset \mathbb{R}^{2}, \quad u=0 \text { on } \partial \Omega .
$$

for the situation depicted in Figure 1. The domain $\Omega$ is partitioned into two
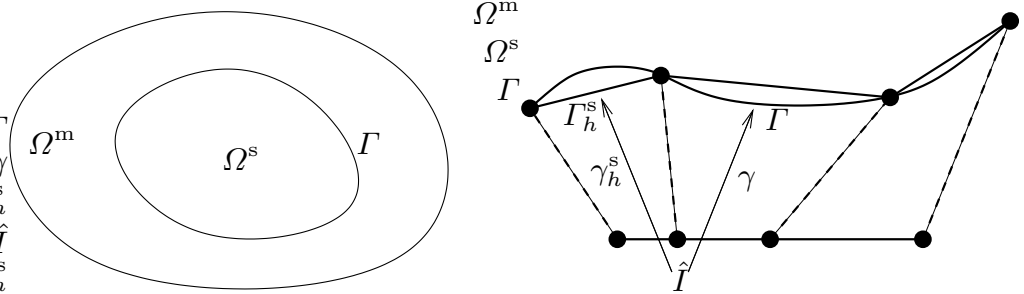

Fig. 1. Left: Decomposition into subdomains $\Omega^{\mathrm{m}}, \Omega^{\mathrm{s}}$. Right: interface $\Gamma$ and its piecewise linear interpolation $\Gamma_{h}^{\mathrm{s}}$.

subdomains $\Omega^{\mathrm{m}}$ and $\Omega^{\mathrm{s}}$ by a sufficiently smooth curve $\Gamma$ of length $L$, given in terms of an arc length parametrization $\gamma: \hat{I} \rightarrow \Gamma, \quad \hat{I}=[0, L)$. By introducing the spaces $X=H_{*}^{1}\left(\Omega^{\mathrm{m}}\right) \times H_{*}^{1}\left(\Omega^{\mathrm{s}}\right)$ and $M=H^{-1 / 2}(\Gamma)$, with $H_{*}^{1}\left(\Omega^{\mathrm{i}}\right)$ respecting the Dirichlet conditions on $\partial \Omega, i=\mathrm{m}, \mathrm{s}$, the boundary value problem (1) can be transformed into the following saddle point problem: find $(u, \lambda) \in X \times M$ such that

$$
\begin{aligned}
a(u, v)+b(v, \lambda) & =f(v), & & v \in X, \\
b(u, \mu) & =0, & & \mu \in M,
\end{aligned}
$$

with the obvious meanings for $a(\cdot, \cdot)$ and $f(\cdot)$, and with the coupling bilinear form $b(\cdot, \cdot)$ given by

$$
b(v, \mu)=\langle[v], \mu\rangle_{\Gamma}, \quad(v, \mu) \in X \times M,
$$

where [.] denotes the jump across $\Gamma$. The discretization of $\Omega$ by $\Omega^{\mathrm{s}}$ and $\Omega^{\mathrm{m}}$ with simplicial triangulations results in piecewise linearizations $\Gamma_{h}^{\mathrm{s}}$ and $\Gamma_{h}^{\mathrm{m}}$ of the curved interface $\Gamma$, given by piecewise linear parametrizations $\gamma_{h}^{\mathrm{s}}: \hat{I} \rightarrow \Gamma_{h}^{\mathrm{s}}$ and $\gamma_{h}^{\mathrm{m}}: \hat{I} \rightarrow \Gamma_{h}^{\mathrm{m}}$, respectively. These parametrizations enable us to uniquely identify each point on $\Gamma_{h}^{\mathrm{m}}$ with a point on $\Gamma_{h}^{\mathrm{s}}$, providing a projection operator

$$
P_{\mathrm{s}}:\left(L^{2}\left(\Gamma_{h}^{\mathrm{m}}\right)\right)^{2} \rightarrow\left(L^{2}\left(\Gamma_{h}^{\mathrm{s}}\right)\right)^{2}, \quad v_{\mathrm{m}} \mapsto P_{\mathrm{s}} v_{\mathrm{m}}=v_{\mathrm{m}} \circ \gamma_{h}^{\mathrm{m}} \circ\left(\gamma_{h}^{\mathrm{s}}\right)^{-1} .
$$

In order to obtain an approximate coupling bilinear form $b_{h}(\cdot, \cdot)$, we introduce a mesh dependent jump over the interface grid $\Gamma_{h}^{\mathrm{s}}$ by 


$$
[v]_{h}=v_{\mathrm{s}}-P_{\mathrm{s}} v_{\mathrm{m}} .
$$

The approximation $M_{h}$ of $M$ is given by one of the common discrete Lagrange multiplier spaces on $\Gamma_{h}^{\mathrm{s}}$, see e.g. $[2,3,8,5]$. The space $X$ is approximated by $X_{h}$ using $P 1$ finite elements. We define $b_{h}(\cdot, \cdot)$ in terms of $[\cdot]_{h}$ by

$$
b_{h}(v, \mu)=\left([v]_{h}, \mu\right)_{L^{2}\left(\Gamma_{h}^{\mathrm{s}}\right)}, \quad(v, \mu) \in X_{h} \times M_{h} .
$$

Approximating $a(\cdot, \cdot)$ and $f(\cdot)$ by $a_{h}(\cdot, \cdot)$ and $f_{h}(\cdot)$, we obtain the discrete saddle point problem of finding $\left(u_{h}, \lambda_{h}\right) \in X_{h} \times M_{h}$ as the solution of

$$
\begin{aligned}
a_{h}\left(u_{h}, v\right)+b_{h}\left(v, \lambda_{h}\right) & =f_{h}(v), & & v \in X_{h}, \\
b_{h}\left(u_{h}, \mu\right) & =0, & & \mu \in M_{h} .
\end{aligned}
$$

For an analysis of (6), we refer to [4]. There, in order to obtain a priori bounds for the discretization error, we proceed in two steps. In the first step, we introduce and analyze a new discrete variational problem based on blending elements, where the curved interfaces are resolved exactly, see [6]. In the second step, we interpret (6) as a perturbed blending approach, and estimate the perturbation terms obtained from the first Strang lemma. The main result is:

Theorem 1. Let $(u, \lambda)$ and $\left(u_{h}, \lambda_{h}\right)$ solve (2) and (6), respectively. Then

$$
\left\|u-u_{h}\right\|_{X_{h}}+\left\|\lambda-\lambda_{h}\right\|_{M} \leq C(u) \max _{i=m, s} h_{i} .
$$

In [4], several numerical tests in $2 \mathrm{D}$ are provided to verify the theoretical results. Here, we focus on a 3D example. An exact parametrization of the interface $\Gamma$ is often not available. Therefore, an alternative definition of the projection operator $P_{\mathrm{S}}$ from (4) is required. This can be achieved for each slave element side by using the piecewise constant normal projection of the corresponding master sides, [9]. We remark that the analysis above has to be extended to this case in order to handle the lack of regularity of $P_{\mathrm{s}}$. For the following example, we use this alternative projection operator to define the coupling bilinear form $b_{h}(\cdot, \cdot)$.

For the domain $\Omega$, a ball of radius 0.9 is cut out of a concentric ball of radius 1.1. The subdomains $\Omega_{1}$ and $\Omega_{2}$ are the parts of $\Omega$ with radii greater and less than 1, respectively, their common interface $\Gamma$ being the unit sphere. The exact solution depends only on the radius $r$ and is set to be $u(r)=a r^{-2}+$ $b r$ with $a, b$ chosen such that $u$ describes the radial displacement when the domain is subject to a uniform internal pressure of magnitude 1. We exploit the symmetry of the problem data and reduce the computational domain to $\Omega_{r}=\{(x, y, z) \in \Omega: x, y, z>0\}$, adding natural boundary conditions on the symmetry planes. Two initial triangulations with ratios $4: 1$ and $8: 1$ of the number of fine to coarse interface element sides are shown in Figure 2.

In Figure 3, we compare the error decays using different Lagrange multiplier spaces, namely, the standard Lagrange multipliers coinciding with the trace space $W_{h}$ of the $P 1$ finite element functions on $\Omega_{h}^{\mathrm{s}}$, with the dual 

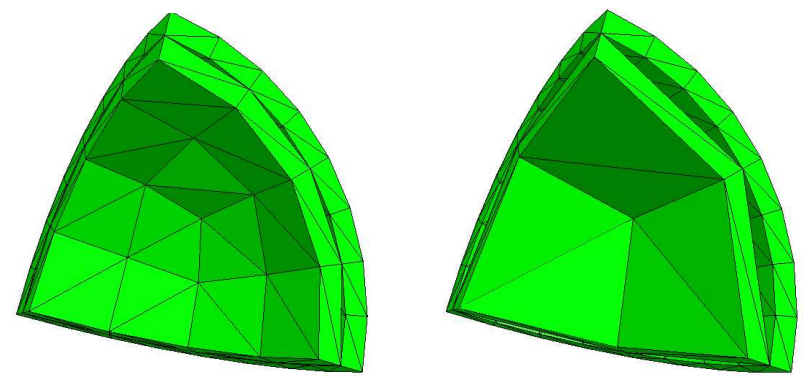

Fig. 2. Initial triangulations: ratios $4: 1$ and $8: 1$.
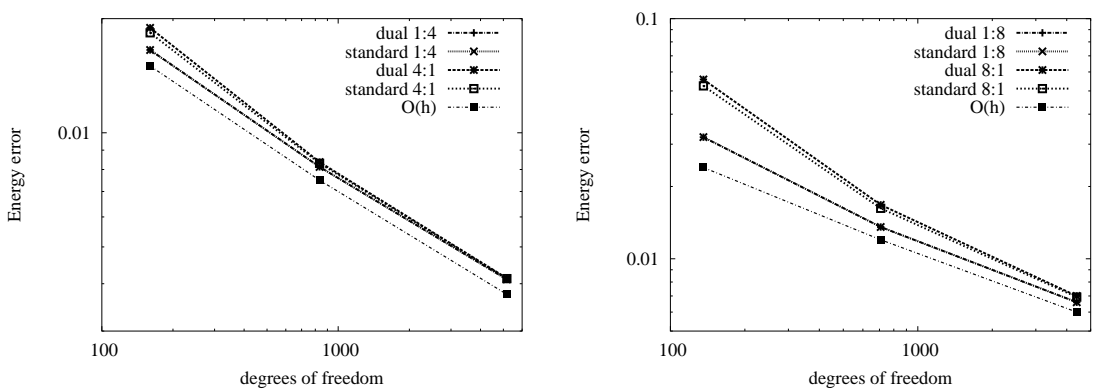

Fig. 3. 3D example: error decay using different Lagrange multiplier spaces.

ones spanned by piecewise linear discontinuous basis functions satisfying a biorthogonality relation with the nodal basis functions of $W_{h}$, see [5]. The choice of the basis functions, either standard or dual, does not greatly influence the numerical results. For very coarse meshes, the use of the coarser grid for the Lagrange multipliers provides better results than the altenative. However, this effect gets small already for very moderate numbers of unknowns.

2D elasticity. We keep the same setting as above and intend to solve (2) with spaces and (bi-)linear forms given by the weak form of the linear elasticity problem of finding a displacement vector field $u$ such that

$$
-\operatorname{div} \sigma(u)=f \text { in } \Omega
$$

supplemented by boundary conditions, by the Saint-Venant Kirchhoff law

$$
\sigma=\lambda(\operatorname{tr} \varepsilon) \mathrm{I}+2 \mu \varepsilon
$$

with the Lamé constants $\mu, \lambda$ and by the linearized strain tensor

$$
\varepsilon(u)=\frac{1}{2}\left(\nabla u+[\nabla u]^{\mathrm{T}}\right) .
$$

We consider the domain visualized in the left picture of Figure 4, see [5]. The ring $\Omega$ with inner radius $r_{\mathrm{i}}=0.9$, outer radius $r_{\mathrm{o}}=1.1$, and moduli $E=1$, 

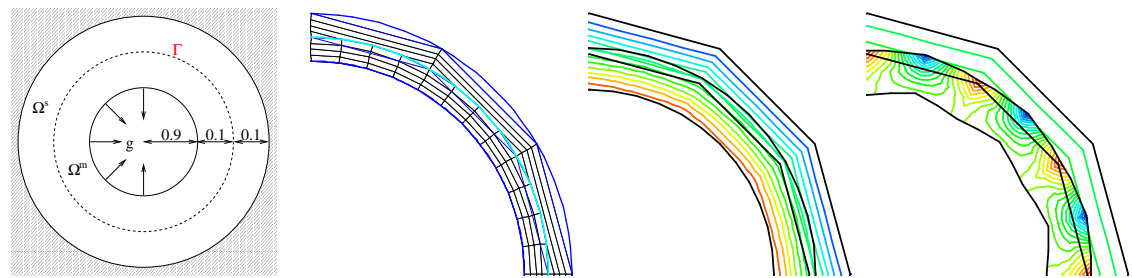

Fig. 4. Model problem, grid, stress using standard and dual multipliers.

$\nu=0.3$, is fixed at the outer boundary, whereas at the inner boundary, a surface traction $f_{\Gamma}(x, y)=-(x, y)^{\mathrm{T}} / r_{\mathrm{i}}$ constant in normal direction is applied. The region is divided into two rings $\Omega^{\mathrm{m}}$ and $\Omega^{\mathrm{s}}$ such that their interface $\Gamma$ is the unit circle. We choose the inner ring to be $\Omega^{\mathrm{m}}$, and the outer ring to be $\Omega^{\mathrm{s}}$. A part of the computational grid is shown in the second picture of Figure 4. The whole grid consists of 240 elements and is constructed in such a way that each element edge on the slave side meets four master edges. Thus, the discrete Lagrange multiplier space $M_{h}$ is defined with respect to the coarse grid on $\Gamma_{h}^{s}$. Again, we compare the standard Lagrange multipliers with the dual ones. In the third and fourth picture of Figure 4, the isolines of the van Mises stresses of the numerical solutions on the deformed domains are plotted. Whereas standard Lagrange multipliers yield a visually satisfying result, the behavior of the solution using dual Lagrange multipliers suffers from strong oscillations along the master interface $\Gamma_{h}^{\mathrm{m}}$.

The misbehavior of the dual Lagrange multipliers, which only occurs preasymptotically and only if they are chosen with respect to the coarser grid, can be explained by the fact that quantities constant in normal or tangential direction are not transferred correctly between the two grids. In [5], we introduce and analyze a modification curing this misbehavior, and at the same time preserving the advantages of the dual approach. We modify $b_{h}(\cdot, \cdot)$ in $(5)$ to

$$
b_{h}^{\bmod }\left(v_{h}, \mu_{h}\right)=\int_{\Gamma_{h}^{\mathrm{s}}} \mu_{h} v_{\mathrm{s}}-\mu_{h}^{\bmod } P_{\mathrm{s}} v_{\mathrm{m}}, \quad v_{h} \in X_{h}, \mu_{h} \in M_{h},
$$

where we replace $\mu_{h}$ for the coupling to the master side by $\mu_{h}^{\bmod }=\mu_{h}+\Delta \mu_{h}$. The modification $\Delta \mu_{h}$ of a discrete Lagrange multiplier $\mu_{h} \in M_{h}$ is defined edgewise on the elements of the interface grid $\Gamma_{h}^{\mathrm{s}}$, see [5]. There, we show that the resulting discrete problem $(6)$ with $b_{h}(\cdot, \cdot)$ replaced by $b_{h}^{\bmod }(\cdot, \cdot)$ has the following properties: a diagonal matrix for the coupling on the slave side, symmetry, preservation of linear momentum, reduction to the unmodified dual approach in case of straight interfaces, and preservation of quantities constant in normal and tangential direction.

As a numerical test, we compare the error decays using the standard, dual, and modified dual approach. For the left picture of Figure 5, the ratio of slave to master edges is kept constant at 1:4. The modification already improves 

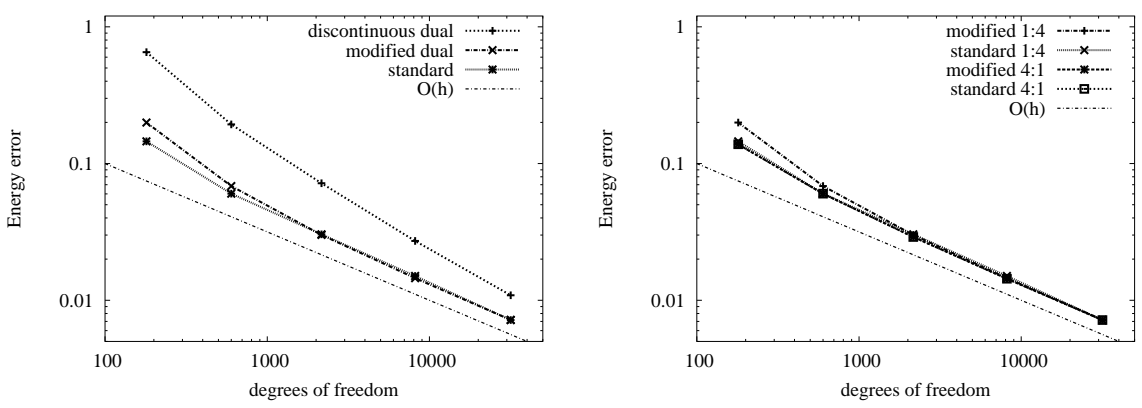

Fig. 5. Left: Decay of the energy error using standard, dual, and modified dual Lagrange multipliers. Right: Change of the Lagrange multiplier side.

the results significantly for a very moderate number of unknowns. We observe that the relative difference in the errors of the unmodified and the modified approach decreases as the number of unknowns increases. This is due to the fact that the modification only enters as a higher order term in the a priori estimates, see [5]. The right picture in Figure 5 illustrates the robustness of the standard and the modified Lagrange multipliers against a change of the master and slave side. We point out that all the benefits of the dual approach are preserved by the modification.

In many applications, symmetry of the domain and the data can be exploited to reduce the problem size. For the example above, we can reduce the computational domain to one quarter $\Omega_{r}=\{(x, y) \in \Omega: x, y>0\}$. On the artificial boundaries $\Sigma_{\xi}=\{(x, y) \in \Omega: \xi=0\}, \xi=x, y$, we have to set appropriate symmetry boundary conditions. For the elasticity setting, these are given by homogeneous Dirichlet data in the normal and homogeneous Neumann data in the tangential direction. In the framework of mortar methods, this would require us to handle the nodes $p_{x}=(1,0)^{\mathrm{T}}$ and $p_{y}=(0,1)^{\mathrm{T}}$ belonging to the triangulation on $\Omega_{r}^{\mathrm{s}}$ as crosspoints for the normal and as usual slave nodes for the tangential components. Since this can be a tedious task to realize during the matrix assembly in existing codes, we suggest to use a simple manipulation of the saddle point system matrix $S=\left(\begin{array}{cc}A & B^{\mathrm{T}} \\ B & 0\end{array}\right)$ for which the nodes $p_{x}, p_{y}$ are handled as usual slave nodes and no Dirichlet conditions are imposed on them. We symmetrically exchange the lines and columns in $B^{\mathrm{T}}$ and $B$ corresponding to the coupling of the Lagrange multipliers in the normal direction of $p_{x}$ and $p_{y}$ to the displacements in the normal direction on the master and slave side by Dirichlet lines and columns. This is exactly the procedure often employed to enforce Dirichlet conditions by means of Lagrange multipliers.

In Figure 6, we test four different approaches. For the calculations leading to the first two pictures, the two Dirichlet lines are inserted in the upper part of $S$. For the first (second) picture, the nodes $p_{x}, p_{y}$ are handled as slave (cross) points in both directions and the Lagrange multiplier space is chosen 

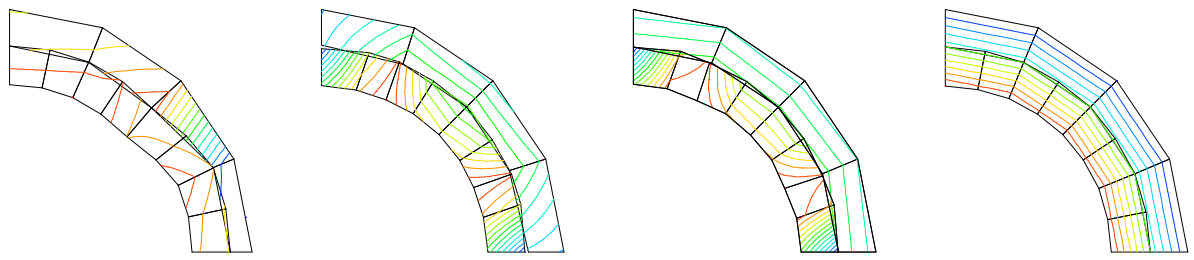

Fig. 6. Handling of symmetry boundaries.

with respect to the finer (coarser) grid. As is expected, both approaches give poor results. For the third picture, we choose the Lagrange multiplier space with respect to the coarser grid, insert only Dirichlet lines in $B$, and keep $B^{\mathrm{T}}$ unchanged. However, this is not enough. This is due to the fact that, in contrast to the full setting, the normal (w.r.t. $\Sigma_{\xi}$ ) components of the Lagrange multipliers in $p_{x}, p_{y}$ are different from zero in the reduced setting on $\Omega_{r}$, since only contributions from $\Omega_{r}$ are assembled. Thus, the master nodes next to $p_{x}, p_{y}$ are subjects to a force pushing in the wrong direction. In order to avoid that these master nodes are affected by the nonzero contribution from the Lagrange multipliers, one also has to insert the corresponding Dirichlet columns in $B^{\mathrm{T}}$, resulting in the right picture of Figure 6 . An equally satisfying result is obtained if the Lagrange multipliers are chosen on the finer grid.

\section{Nonlinear elasticity}

Contact problems. We consider a two-body nonlinear contact problem. The domain $\Omega$ is the union of two initially disjoint bodies $\Omega^{\mathrm{s}}, \Omega^{\mathrm{m}}$, and its boundary $\Gamma=\partial \Omega^{\mathrm{s}} \cup \partial \Omega^{\mathrm{m}}$ is subdivided into three disjoint open sets $\Gamma_{\mathrm{D}}, \Gamma_{\mathrm{N}}, \Gamma_{\mathrm{C}}$. We intend to solve (7)-(9) with Dirichlet and Neumann boundary conditions on $\Gamma_{\mathrm{D}}$ and $\Gamma_{\mathrm{N}}$, respectively, and frictionless Signorini contact conditions on the possible contact boundary $\Gamma_{\mathrm{C}}$, given by

$$
\begin{aligned}
\sigma_{T}\left(u_{\mathrm{s}}\right)=\sigma_{T}\left(u_{\mathrm{m}}\right)=0, & \sigma_{n}\left(u_{\mathrm{m}}\right)([u n]-g)=0, \\
{[u n]-g \leq 0, } & \sigma_{n}\left(u_{\mathrm{m}}\right)=\sigma_{n}\left(u_{\mathrm{s}}\right) \leq 0,
\end{aligned}
$$

where $\sigma_{T}\left(u_{k}\right)$ and $\sigma_{n}\left(u_{k}\right)$ are the tangential part and the normal component of the surface traction $\sigma\left(u_{k}\right) n$, respectively, $k=\mathrm{m}, \mathrm{s}$, and $[u n]$ stands for the jump of the normal displacement across $\Gamma_{\mathrm{C}}$.

We arrive at the problem: find $(u, \lambda) \in X \times M^{+}$such that

$$
\begin{aligned}
a(u, v)+b(v, \lambda) & =f(v), & & v \in X, \\
b(u, \mu-\lambda) & \leq\langle g,(\mu-\lambda) n\rangle_{\Gamma_{\mathrm{C}, \mathrm{s}}}, & & \mu \in M^{+},
\end{aligned}
$$

with $b(v, \mu)=\langle\mu n,[v n]\rangle_{\Gamma_{\mathrm{C}, \mathrm{s}}}$ and $M^{+}=\left\{\mu \in M: \mu_{T}=0,\langle\mu n, v\rangle_{\Gamma_{\mathrm{C}, \mathrm{s}}} \geq\right.$ $0, v \in W, v \geq 0$ on $\left.\Gamma_{\mathrm{C}, \mathrm{s}}\right\}$, where $W$ denotes the trace space of $H_{*}^{1}\left(\Omega^{\mathrm{s}}\right)$ restricted to $\Gamma_{\mathrm{C}, \mathrm{s}}$ and $M$ is its dual. We use standard piecewise linear finite 
elements for $X$ and discontinuous piecewise linear dual Lagrange multipliers for $M$. The discrete convex cone $M_{h}^{+}$is defined with respect to the scalar dual basis funtions $\psi_{i}$ as

$$
M_{h}^{+}=\left\{\mu_{h} \in M_{h}: \mu_{h}=\sum \alpha_{i} \psi_{i}, \alpha_{i} \in \mathbb{R}^{2}, \alpha_{i} n \geq 0, \alpha_{i} \times n=0\right\} .
$$

In [7], optimal a priori error bounds are obtained for the correspondig discrete problem formulation. Concerning the numerical solution process, we employ a primal-dual active set strategy (PDASS) in order to deal with the nonlinearity of the contact condition (11). Starting from an initial active set, the PDASS checks in each step the sign of the normal stress component for an active node to determine whether the node stays active, and for an inactive node the non-penetration condition to determine whether the node stays inactive. Proceeding like this, a new active set is calculated, and the active nodes provide Dirichlet conditions and the inactive nodes give homogeneous Neumann conditions for the linear system to be solved. The biorthogonality of the dual basis functions spanning $M_{h}^{+}$is of crucial importance for the realization of the PDASS. In particular, the weak formulation of the non-penetration condition, i.e., the third equation of (11), naturally reduces to a pointwise relation which is easy to handle. Moreover, the Lagrange multiplier can be efficiently eliminated yielding a positive definite linear system for the remaining unknowns in each iteration step of the PDASS. Thus, suitable multigrid solvers can be applied. Limiting the maximum number of multigrid iterations per PDASS step yields an inexact strategy.

As a numerical example, we consider the situation depicted in Figure 7. In the left picture, a cross section of the problem definition is shown. The
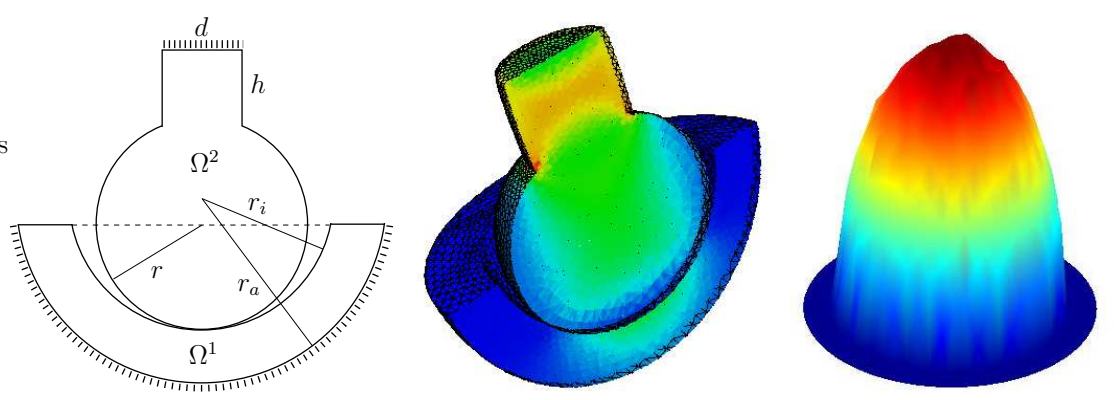

Fig. 7. Problem setting (left), cut through the distorted domains with the effective von Mises stress on level 3 (middle), and the contact stresses $\lambda_{h}$ on level 3 (right).

lower domain $\Omega^{1}$ is the master, and it models a halfbowl which is fixed at its outer boundary. Against this bowl, we press the body modeled by the domain $\Omega^{2}$ which is the slave. At the top of $\Omega^{2}$, we apply Dirichlet data equal to $(0,0,-0.2)^{\top}$. We use $r_{i}=0.7, r_{a}=1.0, r=0.6, h=0.5$ and $d=0.3$, and as 
material parameters, $E_{1}=400 \mathrm{~N} / \mathrm{m}^{2}, \nu_{1}=0.3$ and $E_{2}=300 \mathrm{~N} / \mathrm{m}^{2}, \nu_{2}=0.3$. The second and third picture in Figure 7 show a cut through the domains and the contact stress $\lambda_{h}$ on level 3, respectively.

In Table 1, the exact PDASS is compared with the inexact version. For the

\begin{tabular}{|c|c|c|c|c|c|c|c|c|c|c|}
\hline \multirow{2}{*}{1} & \multirow{2}{*}{$\mathrm{DOF}$} & \multicolumn{5}{|c|}{ exact strategy } & \multicolumn{4}{|c|}{ inexact strategy } \\
\hline & & $K_{l}$ & & $\mid \mathcal{A}$ & $\overline{A_{k}}$ & & $M_{l}$ & & $\left|\mathcal{A}_{k}\right|$ & \\
\hline 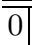 & 312 & 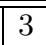 & 0 & 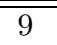 & 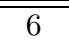 & & 3 & 9 & 6 & \\
\hline 7 & 1623 & 4 & 14 & 26 & 21 & 21 & 4 & $\begin{array}{ll}14 \quad 26\end{array}$ & 22 & 21 \\
\hline 2 & 10062 & 3 & 66 & 88 & 85 & & 3 & $\begin{array}{ll}66 & 91 \\
\end{array}$ & 85 & \\
\hline 3 & 71082 & 4 & 306 & 347 & 336 & 337 & 5 & 306341 & 336 & 336337 \\
\hline
\end{tabular}

Table 1. Comparison between exact and inexact active set strategy.

inexact strategy, we apply only one multigrid step per PDASS iteration. For both strategies, we use a $\mathcal{W}$-cycle with a symmetric Gauß-Seidel smoother with 3 pre- and post-smoothing steps. The second column shows the number of degrees of freedom on level $l$. For the exact strategy, we denote by $K_{l}$ the step in which the correct active set $\mathcal{A}$ is found for the first time, and $M_{l}$ indicates the same quantity for the inexact strategy. By $\left|\mathcal{A}_{k}\right|$, we denote the number of active nodes in iteration $k$ and multigrid step $k$, respectively. They are almost the same, thus, there is no need for solving the resulting linear problems in each iteration step exactly, and the cost of our nonlinear problem is very close to that of a linear problem, given the correct contact zone.

Geometrically nonlinear problems and nonlinear material laws. The validity of the linearized elasticity equations (7)-(9) is restricted to small strains and small deformations. If the strains remain small but the deformations become large, one has at least to consider the geometrically nonlinear elasticity setting. This amounts to using the full Green-St. Venant tensor

$$
E=\frac{1}{2}\left(F^{\mathrm{T}} F-\mathrm{I}\right)=\frac{1}{2}(C-\mathrm{I})
$$

instead of (9), with $F=\mathrm{I}+\nabla u$ the deformation gradient and $C=F^{\mathrm{T}} F$ the right Cauchy-Green strain tensor. We keep the constitutive law (8) as

$$
S=\lambda(\operatorname{tr} E) \mathrm{I}+2 \mu E=\mathcal{C} E,
$$

defining the second Piola-Kirchhoff stress tensor $S$, with $\mathcal{C}$ the Hooke-tensor. We solve

$$
-\operatorname{div}(F S)=f
$$

complemented by appropriate boundary conditions. In the weak setting, this gives the linear form $a(u, \cdot)$ given by $a(u, v)=\sum_{i=1}^{4} a_{i}(u, v)$, where 


$$
\begin{array}{ll}
a_{1}(u, v)=\int_{\Omega} \mathcal{C} \varepsilon(u): \varepsilon(v) d x, & a_{2}(u, v)=\frac{1}{2} \int_{\Omega} \mathcal{C}\left[(\nabla u)^{\top} \nabla u\right]: \nabla v d x, \\
a_{3}(u, v)=\int_{\Omega} \nabla u \mathcal{C} \nabla u: \nabla v d x, & a_{4}(u, v)=\frac{1}{2} \int_{\Omega} \nabla u \mathcal{C}\left[(\nabla u)^{\top} \nabla u\right]: \nabla v d x .
\end{array}
$$

Still, the applicability of (13)-(15) is limited to small strains. In order to extend the model to large strains, we have to introduce another kind of nonlinearity by means of nonlinear material laws. In particular, to solve (15), we employ the Neo-Hooke law given by

$$
S=\mu\left(\mathrm{I}-C^{-1}\right)+\frac{\lambda}{2}\left(J^{2}-1\right) C^{-1},
$$

with $J=\operatorname{det}(F)$ denoting the determinant of the deformation gradient. While in (13) the nonlinearity enters in terms of polynomials of $\nabla u$, it is given in terms of its inverse in (16).

Despite the complexity of the nonlinear setting, the subdomain coupling via Lagrange multipliers remains the same as for linear problems. In order to calculate a numerical solution, we eliminate the discrete Lagrange multipliers and apply a Newton iteration to the constrained problem. We note that this elimination is very efficient when we use the dual basis functions for spanning the Lagrange multiplier space. Moreover, the Jacobian of the constrained system is positive definite and admits the use of multigrid solvers for the linear system in each Newton step.

For a first numerical test, we consider a square $\Omega=(0,1)^{2}$, decomposed into four quadrilaterals $\Omega_{i j}=((i-1) / 2, i / 2) \times((j-1) / 2, j / 2), i, j=1,2$. The material parameter are set to $E=2000 \mathrm{~N} / \mathrm{m}^{2}, \nu=0.4$ on $\Omega_{11}, \Omega_{22}$ and to $E=300 \mathrm{~N} / \mathrm{m}^{2}, \nu=0.3$ on $\Omega_{21}, \Omega_{12}$. We use the linear elasticity model on $\Omega_{11}, \Omega_{22}$, and the nonlinear Neo-Hooke model on $\Omega_{21}, \Omega_{12}$. The domain is fixed at its upper and lower boundary segment, whereas on the left and right segment, a force density of magnitude $10+y(y-1)$ pointing inside the domain is applied. The first two pictures of Figure 8 show the deformed grids with deformations magnified by a factor 100 for two ways of dealing with the crosspoint $p^{\mathrm{c}}=(1 / 2,1 / 2)$. In the first calculation, the crosspoint is left free
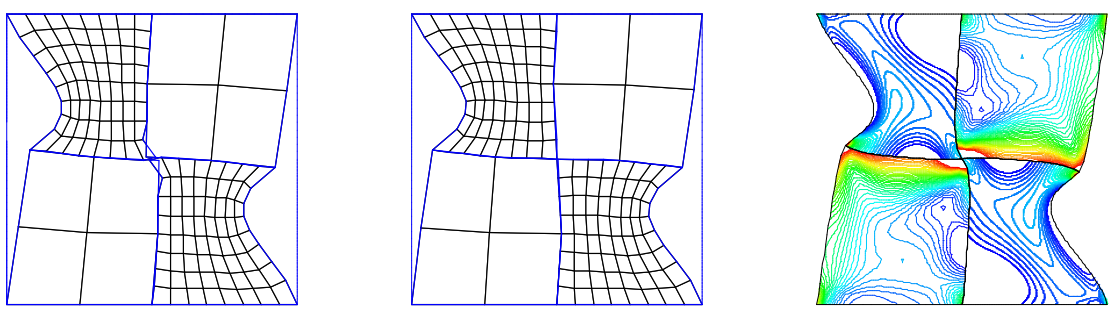

Fig. 8. Deformations without (left) and with (middle) continuity requirement. 
leading to unphysical penetrations of the subdomains. In contrast, for the second calculation, continuity is enforced; cf. [2]. We note that the undesired effect of the first calculation diminishes when the meshsize is reduced.

As 3D example, we consider an I-beam as illustrated in Figure 9. The beam
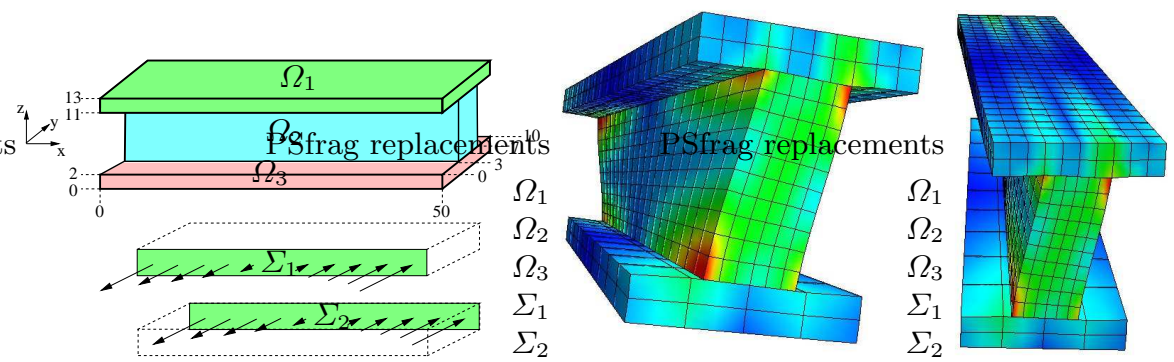

Fig. 9. Left: I-beam decomposed into three subdomains and urface forces on $\Sigma_{1}, \Sigma_{2} \subset \partial \Omega_{1}$. Middle and right: deformed beam.

is decomposed into three subdomains $\Omega_{1}:=(0,50) \times(0,10) \times(11,13), \Omega_{2}:=$ $(0,50) \times(3,7) \times(2,11)$ and $\Omega_{3}:=(0,50) \times(0,10) \times(0,2)$. On all subdomains, we consider as material parameters $E=100, \nu=0.3$. The beam is fixed in all directions on the plane $x_{3}=0$, and in $x_{3}$-direction on the plane $x_{3}=13$. On $\Sigma_{1}, \Sigma_{2} \subset \partial \Omega_{1}$ with $\Sigma_{1}=(0,50) \times\{0\} \times(11,13), \Sigma_{2}=(0,50) \times\{10\} \times(11,13)$, surface forces $f(x)=-2+4 x / 50$ in $y$-direction are applied.

In the middle and the right picture of Figure 9, the deformed beam is plotted using the Neo-Hooke law on all subdomains. We note that we do not require the subdomain triangulations to match across their common interfaces; we can employ different meshsizes and uniformly structured grids as well as different models on each subdomain. The deformed grid suggests that we can employ the fully linearized one for the lower subdomain $\Omega_{3}$, where only small displacements and strains occur, the geometrically nonlinear one for the upper part $\Omega_{1}$ because of large displacements but small strains, and the Neo-Hooke law for the middle beam $\Omega_{2}$ with both large deformations and strains.

To justify our strategy, we compare the use of different models on the individual subdomains. We indicate a configuration by $i j k, i, j, k \in\{l, g, n\}$, where $l, g$ and $n$ stand for linear, geometrically nonlinear and Neo-Hooke, respectively, and the position indicates the corresponding subdomain. In Figure 10 , the displaments in $x_{1}$-direction along the line $(0,50) \times\{3\} \times\{11\}$ on $\Omega_{1}$ are plotted for several different settings. In the left picture, the solid, dashed, and dash-dotted lines correspond to the models $n n n, l l l$, and $g g g$, respectively. Whereas the linear model is symmetric with respect to $x_{1}^{*}=25$, the nonlinear ones exhibit a rather unsymmetric and more realistic behavior. Moreover, on each line, the markers indicate the results when the model on the lower subdomain $\Omega_{3}$ is switched. There is no visible difference between using the linear or the nonlinear relationship. In the right picture, we primarly compare 

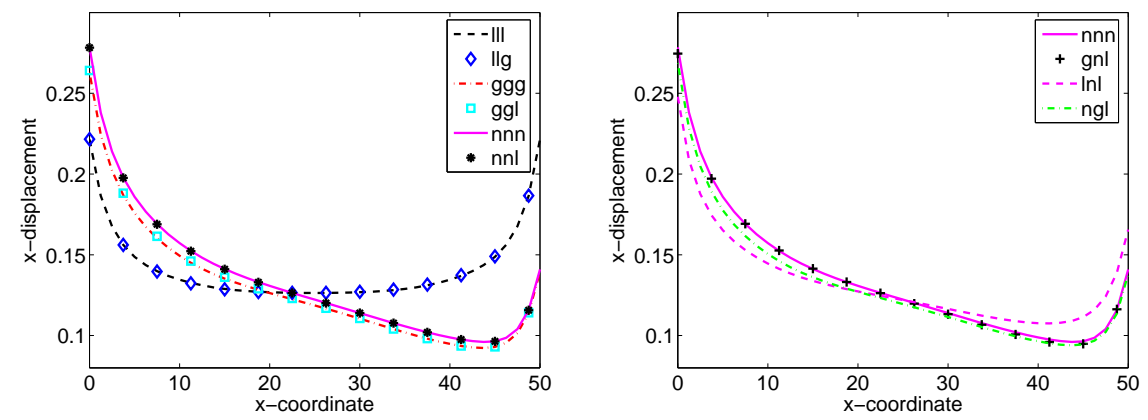

Fig. 10. Comparison of varying model equations in the subdomains.

configurations $n n n$ and $g n l$, where no real difference can be observed. The results for $n g l$ and $l n l$ in combination with the left picture indicate that it is necessary to use the Neo-Hooke law on the middle subdomain $\Omega_{2}$, while on the upper part $\Omega_{1}$, the geometrically nonlinear model is required.

\section{References}

1. F. Ben Belgacem. The mortar finite element method with Lagrange multipliers. Numer. Math., 84(2):173-197, 1999.

2. C. Bernardi, Y. Maday, and A. T. Patera. A new nonconforming approach to domain decomposition: the mortar element method. In Nonlinear partial differential equations and their applications. Collège de France Seminar, Vol. XI (Paris, 1989-1991), volume 299 of Pitman Res. Notes Math. Ser., pages 13-51. Longman Sci. Tech., Harlow, 1994.

3. D. Braess and W. Dahmen. Stability estimates of the mortar finite element method for 3-dimensional problems. East-West J. Numer. Math., 6(4):249-263, 1998.

4. B. Flemisch, J. Melenk, and B. Wohlmuth. Mortar methods with curved interfaces. Appl. Numer. Math., in press, 2005.

5. B. Flemisch, M. Puso, and B. Wohlmuth. A new dual mortar method for curved interfaces: 2D elasticity. Internat. J. Numer. Methods Engrg., in press, 2005.

6. W. J. Gordon and C. A. Hall. Transfinite element methods: blending-function interpolation over arbitrary curved element domains. Numer. Math., 21:109-129, $1973 / 74$.

7. S. Hüeber, M. Mair, and B. Wohlmuth. A priori error estimates and an inexact primal-dual active set strategy for linear and quadratic finite elements applied to multibody contact problems. Appl. Numer. Math., in press, 2005.

8. C. Kim, R. D. Lazarov, J. E. Pasciak, and P. S. Vassilevski. Multiplier spaces for the mortar finite element method in three dimensions. SIAM J. Numer. Anal., 39(2):519-538, 2001.

9. M. Puso. A 3D mortar method for solid mechanics. Internat. J. Numer. Methods Engrg., 59(3):315-336, 2004. 